\title{
Simulation of Optical Excitation to and Emission from Electron Fabry-Perot States Subject to Strong Inelastic Scattering.
}

\author{
LEONARD F. REGISTER \\ Beckman Institute and Coordinated Science Laboratory University of Illinois at Urbana Champaign, 61801

\begin{abstract}
Photon induced carrier excitation to and escape from Fabry-Perot electron states in the presence of subsequent inelastic scattering is simulated. The Fabry-Perot states exist above a quantum well sandwiched between quarter-wave stacks of potential wells and barriers. Two numerical methods are used: simulation of inelastic scattering via absorbing (optical) potentials, and simulation via Schrödinger Equation Monte Carlo.
\end{abstract}

Keywords: quantum transport, inelastic scattering, polar-optical phonon scat tering, Fabry-Perot electron states, electron Bragg reflectors

Recently, localized electron states have been observed at energies above the potential barriers in the infrared absorption spectra of semiconductor semiconductor quantum wells sandwiched between quarter-wave stacks of potential barriers and wells [1,2]. For strong spatial confinement by the "electron Bragg reflectors," inelastic scattering becomes the dominate path by which electrons can leave these "Fabry-Perot" states, so that understanding this latter process is crucial to interpretation of the experimental results. To this end, two numerical methods for simulating quantum transport in the presence of such strong inelastic scattering are applied: simulation of inelastic scattering via absorbing (optical) potentials [3,4], and simulation via "Schrödinger Equation Monte Carlo" (SEMC) $[4,5]$.

Figure 1 shows the potential and effective mass functions used within an effective mass approximation in this work to model the AlInAs-GaInAs system of Ref. [1], and the resulting subband energy minimums. To model the intersubband excitation of elec- trons from the ground subband states to Fabry-Perot states due to absorption of light, these states are coupled via the operator $A_{x} \partial / \partial x$ and the ground state probability densities are held constant. Here $A_{x}$ is the well normal component of the vector potential.

In the absorbing potential model, inelastic scattering and the resulting loss of phase coherence are modeled via the introduction of an imaginary potential function $-i R \hbar / 2$ within Schrödinger's equation, where $R$ is the scattering rate [4]. Figure 2(a) and (b) show simulated optical absorption (electron excitation) and electron emission (escape) probabilities, respectively, for the number of well-barrier pairs ranging from zero to five. A uniform scattering rate in the Fabry-Perot states of $\mathrm{R}=1.5 \times 10^{13} \mathrm{sec}^{-1}$ provided good agreement to the absorption spectra measured in Ref. [1] at 10K. For this figure the well-parallel momenta was fixed to zero to isolate the effects of scattering. However, averaging over the well-parallel momentum states consistent with the sheet carrier density of $3 \times 10^{11}$ $\mathrm{cm}^{-2}\left(1 \times 10^{18} \mathrm{~cm}^{-3}\right)$ in the experiments had little 


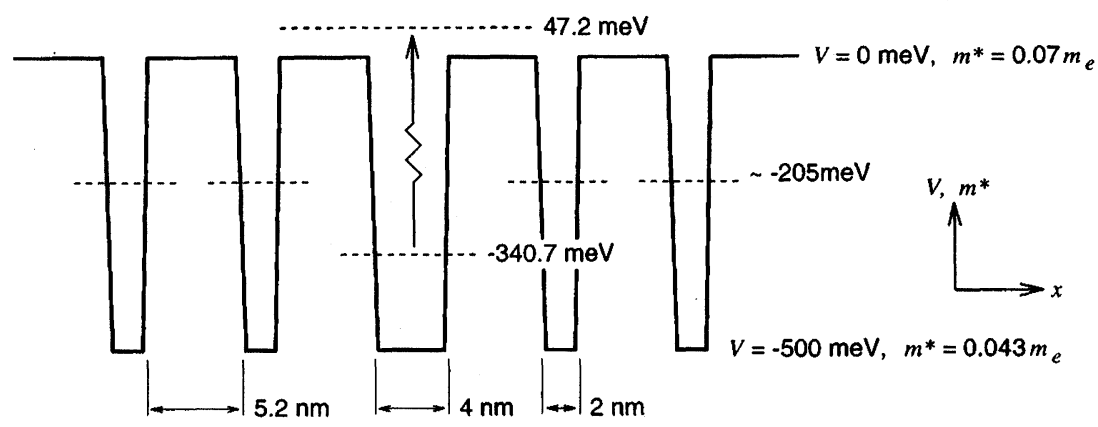

FIGURE 1 Potential and effective mass functions used in this work to model the system of Ref. [1] along with the resulting energy subband minimums. A $0.4 \mathrm{~nm}$ grid is used for discretization of Schrödinger's equation

effect on the simulation results. As can be seen, the excitation probability vs. photon energy quickly saturates with the number of barrier well-pairs to a broadening determined by the inelastic scattering rate, while the emission probability continues to decrease exponentially despite any dephasing within the Bragg reflectors. Also note that there is no emission until the photon plus ground-state energy exceeds the barrier height despite the apparent broadening, as required for energy conservation.

A more rigorous approach to modeling dissipative quantum transport is provided by SEMC [3,4]. In brief, for carrier-phonon interactions, a set of Schrödinger equations for the carrier is solved simultaneously, with the individual equations corresponding to the initial or "trunk" state (here a Fabry-Perot state) and various final or "branch" states of the phonon system (here subdivided by well-parallel momentum), with energies separated by plus or minus the phonon energy for phonon absorption and emission, respectively. Non-local coupling potentials between the trunk and branch states are obtained by Monte Carlo sampling of (the spatial correlation functions of) the true carrier-phonon interactions, as described in detail in Ref. [4]. The same absorbing potential used in the previous section was included in these branch states to represent still later scattering.

Figure 3 shows the calculated absorption probability at $0 \mathrm{~K}$ in the presence of polar-optical-phonon scat-
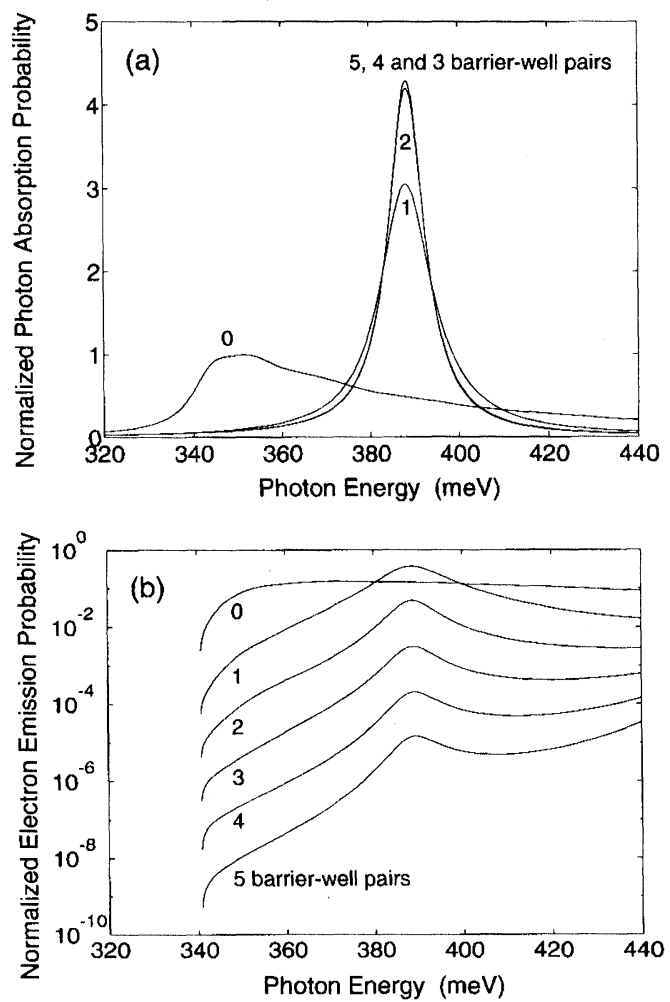

FIGURE 2 (a) Electron excitation (optical absorption) and (b) escape (emission) probabilities, respectively, obtained using the absorbing potential approach 
tering only, for zero and five well-barrier pairs. The phonon energy, $30.2 \mathrm{meV}$, and coupling constants where based on InAs material parameters only, for simplicity. The parabolic and single-phonon-band approximations used here notwithstanding, this result demonstrates that polar-optical phonon scattering is insufficient to produce the experimentally observed broadening. The large electron momentum change required to scatter back to the ground-state subband provides a "bottleneck" to the polar optical phonon scattering process. With $1 \times 10^{18}$ electrons $/ \mathrm{cm}^{3}$, the additional observed broadening is likely do to a combination of carrier-carrier scattering and interface roughness and impurity induced local variations in the bound state energies. However, this simulated broadening still represents multi-order-of-magnitude variations in the trunk state electron probability density from that of the scattering-free electron system; i.e., this scattering is far from perturbative. Variations in the electron excitation and emission probabilities with the number of barrier-well pairs are qualitatively similar to those shown in the previous section.

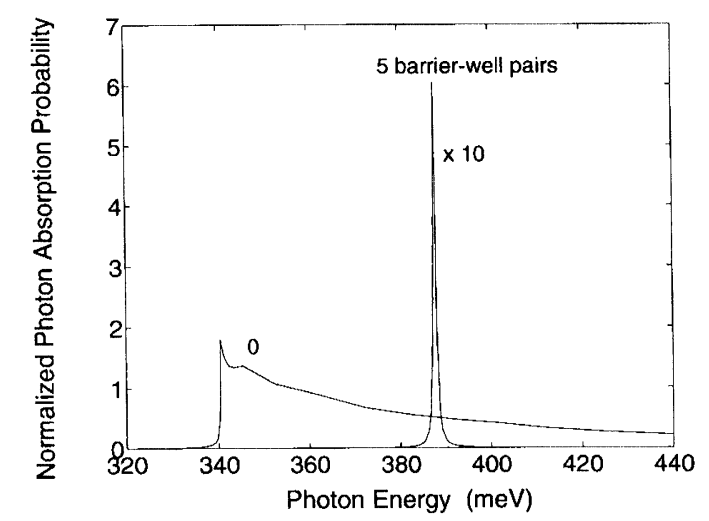

FIGURE 3 Electron excitation probabilities calculated by SEMC for polar-optical-phonon scattering, in the normalized units of Fig. 2
This work exhibits both the effectiveness of electron Bragg reflectors despite dephasive inelastic scattering, and the ability of the numerical methods used to simulate the effects of strong inelastic scattering within mesoscopic structures including simultaneous and proper treatment of both energy broadening effects and energy conservation requirements.

\section{Acknowledgements}

The Author thanks Dr. F. Capasso for suggesting the project and Dr. C. Sirtori and Prof. K. Hess for many useful discussions. This work was supported by the U.S. Army Research Office.

\section{References}

[1] F. Capasso, C. Sirtori, J. Faist, D. L. Sivco, Sung-Nee G. Chu and A. Y. Cho, "Observation of an Electronic Bound State above a potential well," Nature, 358, 565 (13 August 1992).

[2] C. Sirtori, F Capasso, J. Faist, D. L. Sivco, Sung-Nee G. Chu and A. Y. Cho, "Quantum wells with localized states at energies above the barrier height: A Fabry-Perot electron filter," Appl. Phys. Lett. 61, (1994).

[3] See, for example, L. I. Schiff. Quantum Mechanics, McGraw-Hill, 1968, pp. 129-138.

[4] L. F. Register and K. Hess, "Simulation of Carrier Capture in Quantum Well Lasers due to Strong Inelastic Scattering," Proceedings of the Eight International Conference on Superlattices, Microstructures and Microdevices, Cincinnati, August 20-25, 1995, to be published in Superlattices, Microstructures.

[5] L. F. Register and K. Hess, "Numerical Simulation of Electron Transport in Mesoscopic Structures with Weak Dissipation," Phys. Rev. B 49,1900 (1994).

\section{Biography}

Leonard F. Register is a Research Scientist in the Beckman Institute and Coordinated Science Laboratory at the University of Illinois at Urbana-Champaign. His current research interests include dissipative quantum transport, laser simulation, and leakage currents in thin oxides. 

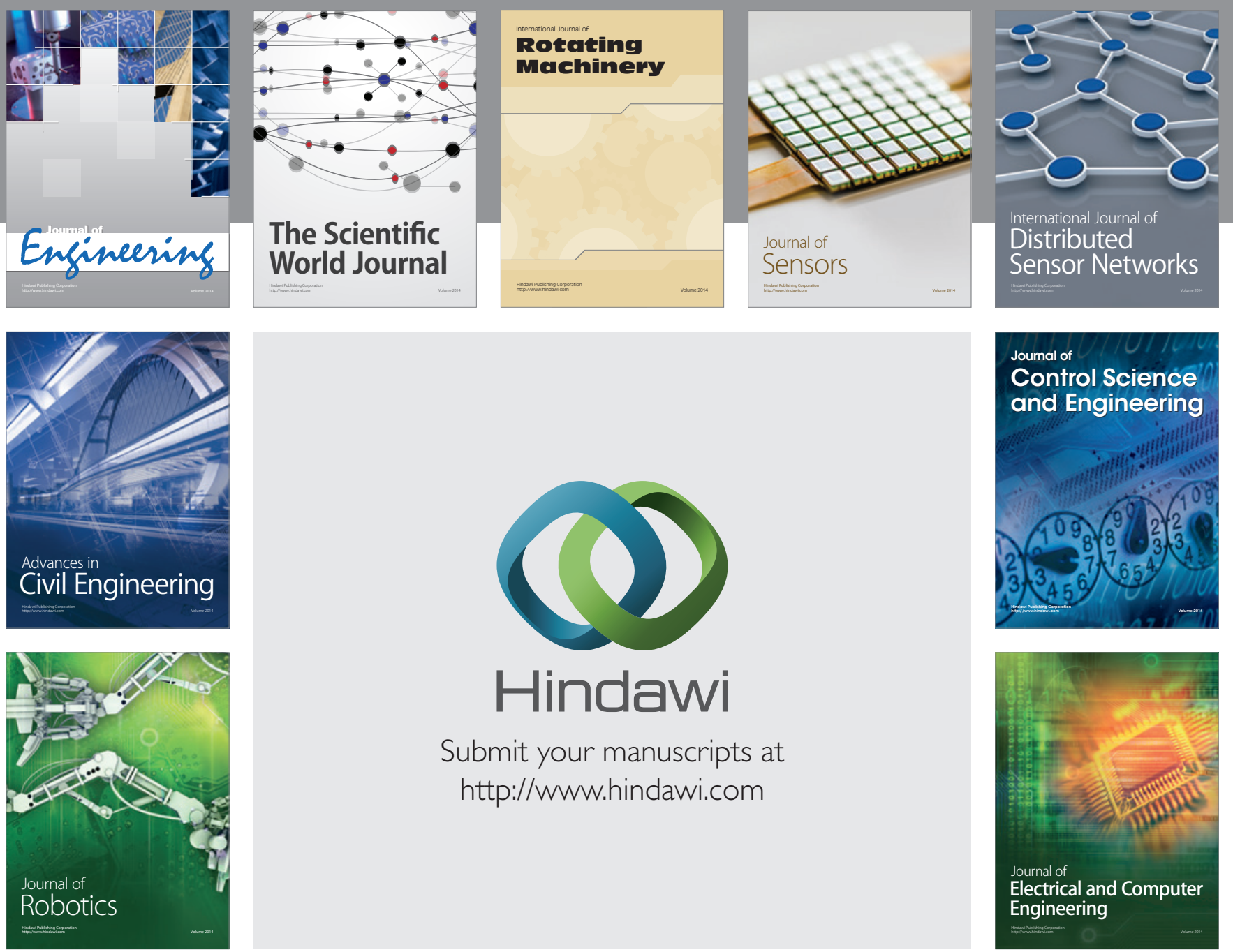

Submit your manuscripts at

http://www.hindawi.com
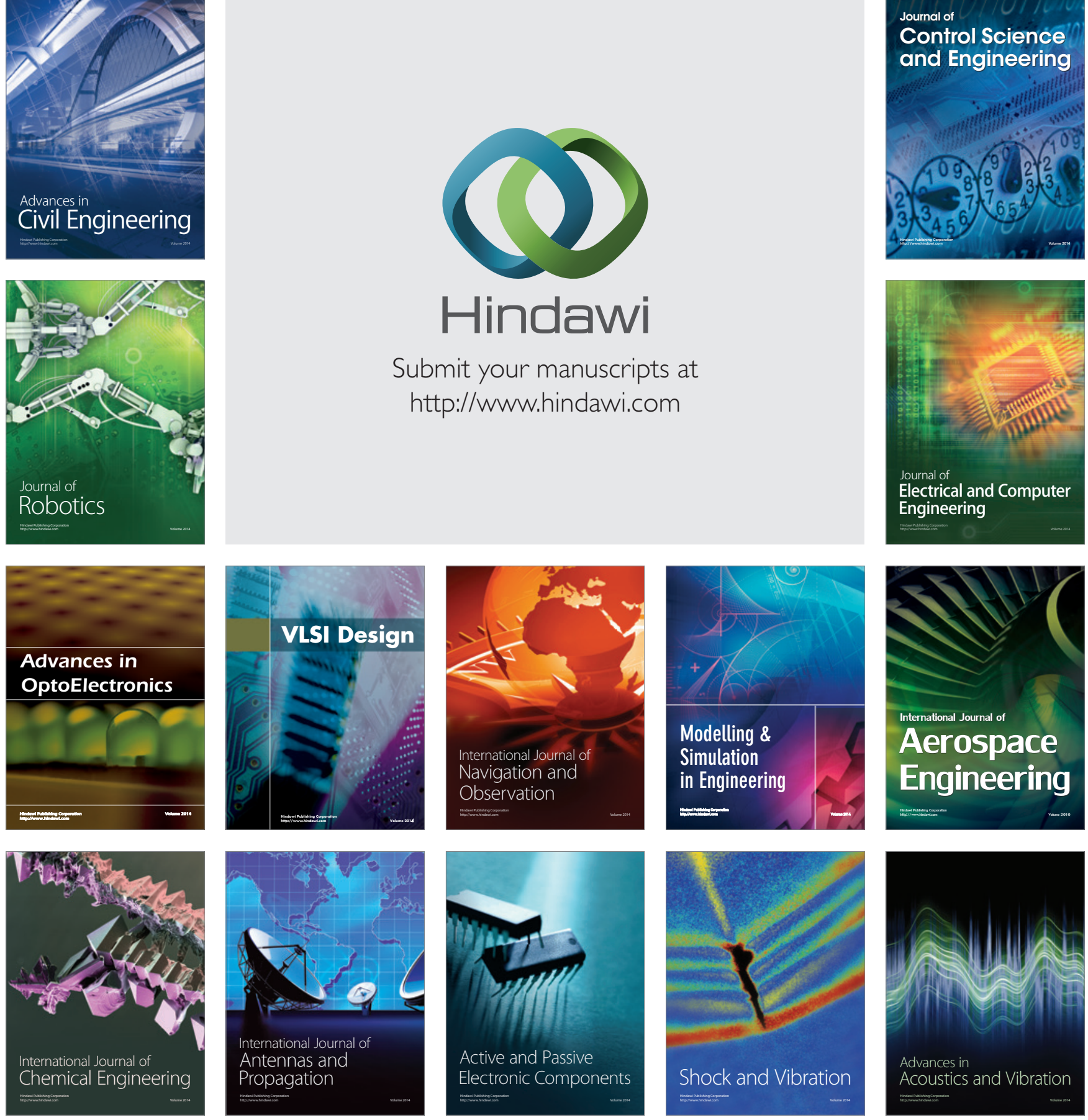